Two developed deficits after surgery. Of 7 with residual epilepsy after surgery, 6 are controlled with AEDs. (Hladky JP et al. Cerebral arteriovenous malformations in children: report on 62 cases. Child's Nerv Syst July 1994;10:328-333).

COMMENT. In reviewing the literature the authors report a postoperative mortality in children ranging from $8.5 \%$ to $11 \%$, versus $23 \%$ to $57 \%$ following conservative management. Surgery is considered the most reliable treatment, combining teams experienced in neurosurgery, embolization, and radiosurgery. The smaller the AVM, the higher the risk of hemorrhage, and the greater the indication for surgery after diagnosis is established. The authors view angiography as superior to CT and MRI in diagnosis and management of AVM.

Postoperative angiography after 2 years is advised to exclude enlarging residual microshunts or recurrence of AVM.

\title{
TRAUMATIC VERTEBRAL ARTERY DISSECTION
}

A girl, aged 9 years, with cerebellar infarction following minor neck injury sustained while ice-skating is reported from West Virginia University, Morgantown, WV. Symptoms began 12 hours after the fall, with vomiting that awakened her from sleep and recurred hourly. She complained of a throbbing occipital headache, stiff neck, photophobia, dizziness on standing, and ataxia. She had horizontal nystagmus, dysmetria bilaterally, and she stood and walked only with support. CT and MRI revealed a cerebellar vermian lesion extending into both hemispheres. Posterior fossa decompression and biopsy showed coagulative necrosis and no neoplasm. Vertebral angiography revealed a traumatic aneurysm within the distal right cervical vertebral artery and recanalization of an embolus in the right posterior inferior cerebellar artery with a narrowed lumen. She was treated with aspirin and recovery was complete after 3 months, with no recurrence at 1 year follow-up. (Sheth RJ, Bodensteiner JB et al. Stroke due to a traumatic vertebral artery dissection in a girl. Clin Pediatr Aug 1994;33:503-505). (respond: Raj D Sheth MD, Dept Neurology, Box 9180, West Virginia University, Health Science Center, Morgantown, WV 26506).

COMMENT. Childhood traumatic vertebral artery stroke was previously thought to affect boys only.(Garg BP et al. Neurology 1993;43:2555). With the increased participation of girls in contact sports, more female cases may be expected.

A further case of vertebral-artery dissection occurring in an 11-yearold boy following a judo session is reported from Besancon, France. (Lannuzel A, Rumbach L et al. Neuropediatrics 1994;25:106-108). CT showed a left thalamic infarct, and angiography revealed fibromuscular dysplasia ("string of beads" lesion) of the left vertebral artery with probable dissection. With anticoagulation, bed rest, and a cervical soft collar, symptoms of headaches, vomiting, left ptosis and diplopia, dysphasia, and ataxia resolved, and the boy was discharged taking aspirin after 2 weeks.

\section{SAFETY OF PHENOBARBITAL IN NEONATES WITH HIE}

Phenobarbital treatment (20 $\mathrm{mg} / \mathrm{kg}$ iv) had no significant effect on cerebral blood flow or blood pressure and heart rate, measured 60 min after a loading dose, in 7 term newborn infants with mild to moderate hypoxic ischemic encephalopathy examined in the Dept of Paediatrics, Alborg Hospital, 
Denmark. Phenobarbital imposed no risk of cerebrovascular damage in newborns with fetal distress. (Andersen $\mathrm{K}$ et al. The effect of phenobarbital on cerebral blood flow in newborn infants with foetal distress. Eur I Pediatr Aug 1994:153:584-587).

COMMENT. Phenobarbital is the most commonly used anticonvulsant in neonates and has been advocated in the prevention of periventricular hemorrhage in preterm infants. This demonstration of the safety of phenobarbital in neonates with HIE is encouraging and should offset in part the poor rating the drug has received in some febrile seizure studies.

\section{RETT SYNDROME}

\section{POLYSOMNOGRAPHY IN RETT SYNDROME}

Respiratory patterns, awake and asleep, were investigated by polysomnography in 30 female patients with Rett syndrome and compared with 30 controls at the Eudowood Division of Pediatric Respiratory Sciences, Johns Hopkins University, and the Department of Neurology, Kennedy Krieger Institute, Baltimore, MD. The median age was 7 years (range, 1 to 32 years). During wakefulness, $67 \%$ of RS patients had a characteristic pattern of disordered breathing (hyperventilation followed by central apnea and desaturation). Breathing was normal during sleep. Arterial oxygen saturation during REM sleep was slightly lower in RS of controls but within normal range. The authors postulate a normal brain-stem control of ventilation in RS and an abnormality or loss of the normal cortical influence on ventilation during wakefulness. The precise cause of the cortical dysfunction is unknown. (Marcus CL, Naidu S et al. Polysomnographic characteristics of patients with Rett syndrome. L Pediatr Aug 1994;125:218-24). (Reprints: Dr Marcus, Johns Hopkins Hospital, Div Pediatric Pulmonology; Park 316, 600 North Wolfe St, Baltimore, MD 21287).

COMMIENT. Patients with Rett syndrome have normal respiration during non-REM sleep, slightly abnormal breathing in REM sleep, and

markedly disturbed breathing during wakefulness. The hyperventilation is attributed to a cortical dysfunction.

\section{RETT VARIANTS: A WIDENING SYMPTOM COMPLEX}

In a collaborative Swedish-Norwegian project, at Goteborg Sweden and Oslo, Norway, a model for the clinical delineation of atypical cases of Rett syndrome was applied to a pilot series of 16 mentally retarded females, aged 11 to 47 years (median: 23). The atypical variants included forme fruste cases (8), late regression (6), and congenital variants (2). The model was based on age $>10$ years, 3 of 6 primary inclusion criteria for RS (eg. hand stereotypies, decelerated head growth, stages of regression and recovery of contact), and 5 of 11 supportive characteristics (eg. breathing irregularities, air swallowing, teeth grinding, gait dyspraxia, scoliosis). The model identified RS variants and distinguished them from other disorders, eg. Angelman's syndrome that fulfilled 3 supportive criteria. (Hagberg BA, Skjeldal OH. Rett variants: a suggested model for inclusion criteria. Pediatr Neurol July 1994;11:5-11). (Respond: Dr Hagberg, Dept of Pediatrics, Ostra Sjukhuset, S-416 85 Goteborg, Sweden).

COMIENT. For a review of recent international research on RS, see "Rett syndrome: from gene to gesture." IR Soc Med Sept 1994;87:562-566). 